\title{
Nanotechnology and mesenchymal stem cells with chondrocytes in prevention of partial growth plate arrest in pigs
}

\author{
Ladislav Planka ${ }^{a}$, Robert Srnec ${ }^{b}$, Petr Rauser ${ }^{b}$, David Stary ${ }^{a}$, Eva Filova ${ }^{d}$, Josef Jancarc, Jana Juhasova ${ }^{e}$, Leos Kren ${ }^{f}$, \\ Alois Necas ${ }^{b}$, Petr Gal
}

Introduction. This study describes the results achieved using a combination of allogeneic mesenchymal stem cells (MSCs) with chondrocytes (CHC) and a new scaffold consisting of type-I collagen and chitosan nanofibers in the prevention of partial growth plate arrest after iatrogenic injury in pigs.

Material and methods. The miniature pig was selected as an experimental model to compare the results in the left femoral bones (MSCs and CHC in scaffold transplantation into the iatrogenic partial distal growth plate defect) and right femoral bones (scaffold alone transplantation). The experimental group consisted of 10 animals. Bone marrow from os ilium as the source of MSCs was used. A porous cylinder consisting of $0.5 \%$ by weight type-I collagen and $30 \%$ by weight chitosan, was the optimal choice. The length of the bone and angular deformity of distal femur after the healing period was measured and the quality and structure of the newly formed cartilage was histologically examined. Results. Transplantation of the composite scaffold in combination with MSCs and chondrocytes led to the prevention of growth disorder and angular deformity in the distal epiphysis of the left femur. Compared to the right (control) femur, tissue similar to hyaline cartilage with signs of columnar organization typical of the growth plate occurred in most cases. Conclusions. The promising results of this study reveal the new and effective means for the prevention of bone bridge formation after growth plate injury.

Key words: mesenchymal stem cells, growth plate defect, cartilage, bone bridge

Received: October 25, 2011; Accepted with revision: April 5, 2012; Available online: June 1, 2012

http://dx.doi.org/10.5507/bp.2012.041

${ }^{a}$ Clinic of Pediatric Surgery, Orthopedics and Traumatology, the Faculty Hospital Brno, Czech Republic

${ }^{b}$ Department of Surgery and Orthopaedics, Small Animal Clinic, Faculty of Veterinary Medicine, University of Veterinary and Pharmaceutical Sciences Brno

cInstitute of Materials Chemistry, Faculty of Chemistry, University of Technology, Brno

dInstitute of Experimental Medicine of the Academy of Sciences of the Czech Republic

eInstitute of Animal Physiology and Genetics of the Academy of Sciences of the Czech Republic

fInstitute of Pathology and Anatomy, Faculty Hospital Brno

Corresponding author: Ladislav Planka, e-mail: Iplanka@seznam.cz

\section{INTRODUCTION}

A growing number of experimental studies are examining ways of exploiting mesenchymal stem cells in the regeneration of injured hyaline cartilage. Earlier experiments raised the question whether it is possible to develop an ideal carrier that could be more easily implanted into a tissue defect and which would meet the requirements for mechanical support and temporary stem cell nutrition. Our study describes the results achieved using a combination of allogeneic mesenchymal stem cells (MSCs) with allogenic chondrocytes $(\mathrm{CHC})$ and a new scaffold consisting of type-I collagen and chitosan nanofibers. The combination of MSCs and CHC drew on the assumption that the differentiation of MSCs would be affected, making it more accurate. Prior to the in vivo implantation, we used the knees of sixty pig cadavers to confirm the procedure of creating a defect in the growth plate and to ensure stable positioning of biocomposite in the defect. The development of a scaffold that would be easier to use was motivated not only by the prospect of more favourable technical characteristics but also increased protection of the transplanted stem cells.

The new collagen carrier manifested significantly better mechanical properties already in the surgical phase of our experiment. The preceding laboratory tests showed the dynamic proliferation and reproduction of mesenchymal stem cells on the scaffold. A comparison of results was made between the experimental and control groups. In terms of preventing growth retardation in the injured extremity and reducing angular deformation of the affected femoral epiphysis, more favourable results had been anticipated.

\section{MATERIAL AND METHODS}

Miniature pigs aged 9 months were selected as our experimental model and purchased, certified breeding from the Institute of Animal Physiology and Genetics of the Academy of Sciences of the Czech Republic in Libechov. The experimental group consisted of 10 animals (left legs - treated group, right legs - control group). 


\section{Cell preparation}

As the source of mesenchymal stem cells we used bone marrow from the os ilium ${ }^{1}$. After aspetic operative field preparation, bone marrow blood was aspirated by a bioptic needle (Iliac-Crest 15G/70-mm bioptic needle (Somatex)) from os ilium (tuber coxae ala osis illii) (Guo et al. 2004) into two $10 \mathrm{ml}$ syringes with $5 \mathrm{ml}$ of PBS (Phosphate Buffered Saline, Dulbecco) + 2\% FBS (Fetal Bovine Serum, StemCell Technologies) and heparin $5 \mathrm{IU} /$ $\mathrm{ml}$. Under sterile conditions, the diluted bone marrow blood (about $20 \mathrm{ml}$ ) was deposited over $15 \mathrm{ml}$ of FPP solution (Ficoll-Paque PLUS, StemCell Technologies).

To separate mononuclear cells from whole bone marrow, we used density gradient centrifugation at $400 \mathrm{~g}$ for $30 \mathrm{~min}$ at room temperature using Ficoll-Paque TM PLUS (StemCell Technologies, Canada). Mononuclear cells in an opalescent layer between Ficoll and blood plasma were removed, washed in a culture medium (see below) and used for propagation under in vitro conditions. The average number of mononuclear cells from each isolation was $25 \times 10^{6}$ cells. Cell number and viability were analyzed on Vi-CELL (Series $\times$ Cell Viability Analyzers) and about 95\% of viable cells were detected. Cells were seeded in tissue culture flasks $\left(1.3 \times 10^{6} / \mathrm{cm}^{2}\right)$ and cultured at $37{ }^{\circ} \mathrm{C}$ in $5 \%$ $\mathrm{CO}_{2}$. The culture medium was $\alpha$ MEM medium (GIBCO, Invitrogen) supplemented with 10\% FBS (Sigma-Aldrich) and Gentamicin (50 mg/ml, Sigma-Aldrich). After $80 \%$ of confluence achievement the adherent MSCs were passaged using trypsin (0.5\% trypsin-EDTA solution, SigmaAldrich) and reseeded at a density of 15,000 cells $/ \mathrm{cm}^{2}$. After the $3^{\text {rd }}$ passage, the MSCs were harvested and analyzed for expression of surface markers CD29, CD44, CD90, CD105 and CD45 by flow cytometry ${ }^{2}$.

The source of chondrocytes was a sample obtained from the non-weight-bearing articular surface of the distal femoral epiphysis in the same pig by bone marrow aspiration (see above) followed by their culturing in a nutrient medium. Cartilage was left immersed in collagenase overnight. The separated chondrocytes were centrifuged at $200 \mathrm{~g}$ for $5 \mathrm{~min}$ and placed in a culture flask with solution of Iscove's Modified Dulbecco's Medium and 15\% Fetal Bovine Serum (FBS), 3 g/1 NaHCO3, 100 IU/ml penicillin, $100 \mu \mathrm{g} / \mathrm{ml}$ streptomycin, $4 \mathrm{mM}$ L-glutamine, $20 \mu \mathrm{g} / \mathrm{ml}$ L-ascorbate-2-phosphate.

The cells were seeded in culture flasks $\left(150 \mathrm{~cm}^{2}\right)$ at a density of approximately $1.3 \times 10^{6}$ cells $/ \mathrm{cm}^{2}$ and cultured at $37{ }^{\circ} \mathrm{C}$ in $5 \% \mathrm{CO}_{2}$. The culture medium was $\alpha$-MEM (Gibco) supplemented with 10\% FBS (Sigma-Aldrich) and Gentamicin ( $50 \mathrm{mg} / \mathrm{ml}$, Sigma- Aldrich). After 24 hours, the non-adherent cells were removed and during the subsequent culturing ( 3 weeks) the medium was exchanged every 3 days. The first colonies of MSCs appeared after 4 to 5 days of culturing and $80 \%$ confluence was achieved after 10 days. The cells were passaged with a solution of $0.5 \%$ trypsin and EDTA (Sigma-Aldrich) for $5 \mathrm{~min}$ at $37^{\circ} \mathrm{C}$ and replated at a density of 15,000 cells $/ \mathrm{cm}^{2}$. For labeling with a fluorescent dye CM-DiI (Molecular Probes) at concentration $5 \mu \mathrm{g} / 2.5 \mathrm{ml}$ PBS, cells were harvested on the day of transplantation, incu- bated for $5 \mathrm{~min}$ at $37^{\circ} \mathrm{C}$ and for $15 \mathrm{~min}$ at $4{ }^{\circ} \mathrm{C}$. The cells were then thoroughly washed in PBS. To induce chondrogenic differentiation ${ }^{3,4}$, the labelled cells were placed in a medium composed of $\alpha$-MEM supplemented with 100 $\mathrm{ng} / \mathrm{ml}$ recombinant human TGF- $\beta_{1}$ (R\&D Systems), 100 $\mathrm{nM}$ dexamethasone (Medochemie), $50 \mu \mathrm{g} / \mathrm{ml}$ L-ascorbic acid 2-phosphate (Sigma-Aldrich) and 1\% ITS (InsulinTransferrin-Selenium-A Supplement, Gibco) for $30 \mathrm{~min}$. Subsequently, the cells were centrifuged ( $700 \mathrm{~g}$ for $5 \mathrm{~min}$ ) and cell pellets were prepared for deposition on the scaffold. After 5 days of culturing in a flask, the cells were passaged and to promote cell reproduction the same medium with $10 \%$ FBS was applied. On achieving confluence, the cells were passaged with trypsin and the second passage was used for seeding the cells. Each scaffold was seeded with $10^{6}$ chondrocytes.

In both cases ( $\mathrm{CHC}$ and MSCs) we used an allogeneic graft. However, we took particular care to ensure that both components came from the same animal.

The experimental procedure was in full agreement with the ethical norms for animal experiments and after agreement by the Ethical Commission (no. 46613/20031020).

\section{Scaffold preparation}

Based on in vitro evaluation performed at the Institute of Experimental Medicine of the Academy of Sciences of the Czech Republic in Prague, a collagen-based scaffold containing chitosan micro- and nanofibers was selected as the most suitable material for meeting specific requirements: the required biological features, stability in simulated body environment, biomechanical response and scaffold behaviour at the site of the planned implantation (ex vivo insertion of the scaffold into an iatrogenic defect in the articular surface and tests of the mechanical properties of the scaffold on prepared pig knee joints). The lyophilization process was optimized in order to obtain interconnected pores of approximately $150 \mu \mathrm{m}$. As a result of successive testing of the suitable composition of a three-dimensional carrier of MSCs, a porous cylinder consisting of $0.5 \%$ by weight type-I collagen and $30 \%$ by weight chitosan (in proportion to collagen amount), cross-linked by EDC (1-ethyl-3-(3-dimethylaminopropyl) carbodiimide hydrochloride) and catalysed by NHS (N-hydroxysuccinimide 98\%), with chitosan-nanofibersbased structure, seemed to be the optimum choice. To facilitate manipulation during implantation, after lyophilization the cylinder-like substrates were placed such that they could be seeded with cells in a 96-well plate. Prior to implantation, the cylinders were seeded with $2 \times 10^{6}$ allogeneic MSCs and $1 \times 10^{6}$ allogeneic chondrocytes.

\section{Surgical procedure}

Surgery was performed under general anesthesia. All animals were intramuscularly administered tiletaminezolazepam (Zoletil 100, Virbac, France) at $2 \mathrm{mg} / \mathrm{kg}$ dosing, xylazine (Sedazine, Fort Dodge, USA) at $0.5 \mathrm{mg} /$ $\mathrm{kg}$ dosing and ketamine (Ketaset, Fort Dodge, USA) at $2 \mathrm{mg} / \mathrm{kg}$ dosing. All substances were administered 
together in a single syringe. After the start of sedation, i.e. 10 min after administration, an intravenous catheter was inserted in the ear vein and propofol (Propofol 1\%, Fresenius, Austria) was administered at $0.5-1 \mathrm{mg} / \mathrm{kg}$ dosing (depending on effect). The course of anesthesia was consistent with previous experimental projects.

Following all the above mentioned procedures, each pig was subjected to radiological examination of the femur in craniocaudal and lateromedial projection. The animal was placed on the operating table in the right lateral position and after sufficient exposure to disinfectant solution, draping of the operative field followed including the placement of the protective sheet. Lateral arthrotomy was performed to reach the left distal end of the femur. The location of the growth zone was confirmed visually. A screw of $4.5 \mathrm{~mm}$ diameter (drill $4.5 \mathrm{~mm}$, SYNTHES) was used to create a defect $12 \mathrm{~mm}$ in depth across the growth plate. After lavage of the defect in the joint, the combined graft consisting of 3 blocks of a collagen-chitosan scaffold, employing allogeneic mesenchymal stem cells in 2 and allogeneic chondrocytes in the middle block, was transferred into the drilled defect (Fig. 1) by press fit fixation. The right combination was obtained by layering 3 biocomposite components: the scaffold and MSCs constituted the first and deepest layer deposited in the defect, the identical scaffold seeded with chondrocytes created the middle section and a scaffold with MSCs formed the outer part. To ensure stability of the transplant, the opening was sutured using the edges of periosteum with monofilament fiber (PDS 0, ETHICON). The repositioning of femur in the knee joint was followed by successive suture of arthrotomy in the individual anatomical layers lege artis. Under supervision of an anaesthesiologist, the animal was positioned on the left hip and identical methodology was applied to perform lateral arthrotomy of the right knee joint. A scaffold without mesenchymal stem cells was deposited in the drilled defect. Suture of anatomical layers was performed in the same way as on the left side. Immediately after the surgery, the operative incision was treated with Novikov solution and the experimental animal was placed in a separate box to allow the effects of general anesthesia to subside. Analgesics were used during the follow up period. The observation period of the miniature pigs from implantation to lege artis euthanasia was 16 weeks. During that time we paid particular attention to the cleanliness of the incisions, injury prevention in individual animals and monitoring of possible postoperative complications. At the end of week 16 all animals were euthanized lege artis by intravenous administration of a mixture of embutramide, mebezonium iodide, tetracaine hydrochloride and dimethylformamide (T61, Intervet, the Netherlands) in a dose of $5 \mathrm{ml}$ i.v. pro toto. Following euthanasia, both femurs were excised and placed in $10 \%$ solution of formalin for fixation before histological examination.

\section{Radiological measurements}

Part of our experiment was a comparison of two radiographs. One was taken prior to experimental injury to the

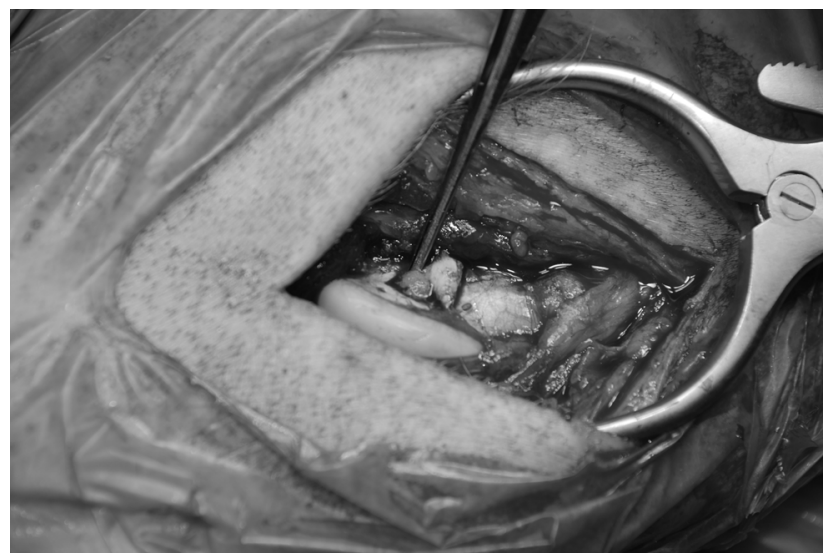

Fig. 1. Implantation of scaffold with MSCs into the defect.

growth zone of the distal end of the femur. The other was taken 16 weeks after the injury. The measurements were performed in craniocaudal projection and a comparison of values obtained from the left (experimental) and right (control) femur was made. Length was measured from the top of the femur head to the most distal point of the femoral condyles. The deformity angle of distal epiphysis of femur was measured as an angle between the longitudinal axis of bone and the tangent intersecting the tops of both condyles.

\section{Histological examination}

All preparates of the distal end of femur placed in fixation solution were transported to the Institute of Pathology and Anatomy of the Faculty Hospital, Brno. Immediately after rinsing off the fixation solution, the bones were immersed in decalcifying Löwy solution which was regularly changed every 2 days. The actual process of decalcification took 10 to 12 days and its completion was followed by preparation of a block of bone tissue containing experimental or control defect in the growth plate. A microtome was used to cut the block into sections $0.2 \mu \mathrm{m}$ thick traversing the specific area and three specimens were prepared: for hematoxylin and eosin staining, fluorescence microscopy and immunohistochemical detection of type-II collagen. In microscopic examination of the specimen stained with hematoxylin and eosin, the coherence of the newly-formed tissue with the surrounding structure of the original growth plate cartilage and the maturity of cartilage in the defect were evaluated. The properties of the newly-created physis were assessed on a scale of 0 to 3 points and modified O'Driscoll score was applied with regard to the renewal of columnar cell formation ${ }^{5-8}$. Immunohistochemical reaction detected type-II collagen fibers and fibre content in the intracellular matrix of the newly-created tissue in the defect of the growth plate. After removal of paraffin from the tissue sections, they were inactivated by endogenous peroxidase $\left(3 \% \mathrm{H}_{2} \mathrm{O}_{2}\right.$ in methanol, $\left.10 \mathrm{~min}\right)$, digested in proteinase $\mathrm{K}$ (10 $\mathrm{min}$ ) and incubated with primary antibody (Novocastra NCL-COLL-IIp, polyclonal, rabbit). 
Table 1. Histological evaluation.

\begin{tabular}{cccc}
\hline & \multicolumn{2}{c}{ Quality of new cartilage } & Col II \\
\cline { 2 - 4 } Final result & O’Driscoll score & $\begin{array}{c}\text { Columnar organization } \\
\text { of CHC }\end{array}$ & negativity \\
\hline 0 & $0-7$ & no & low positivity \\
2 & $8-15$ & no & medium positivity \\
3 & 15 and above & no & high positivity \\
\hline
\end{tabular}

Table 2. Measurements of growth and angle of valgisation in distal epiphysis of femur.

\begin{tabular}{ccccc}
\hline Animal & $\begin{array}{c}\text { Longitudinal growth } \\
\text { of left femur }(\mathrm{cm})\end{array}$ & $\begin{array}{c}\text { Longitudinal growth } \\
\text { of right femur }(\mathrm{cm})\end{array}$ & $\begin{array}{c}\text { Valgisation extent in } \\
\text { distal epiphysis of } \\
\text { left femur }\left(^{\circ}\right)\end{array}$ & $\begin{array}{c}\text { Valgisation extent in } \\
\text { distal epiphysis of } \\
\text { right femur }\left({ }^{\circ}\right)\end{array}$ \\
\hline A1 & 0.5 & 0.4 & 1 & 3 \\
A2 & 0.8 & 0.7 & 2 & 5 \\
A3 & 0.9 & 0.7 & 1 & 6 \\
A4 & 0.7 & 0.3 & 2 & 5 \\
A5 & 0.6 & 0.5 & 2 & 4 \\
A6 & 0.5 & 0.6 & 1 & 5 \\
A7 & 0.7 & 0.4 & 2 & 3 \\
A8 & 0.8 & 0.6 & 1 & 5 \\
A9 & 0.9 & 0.8 & 2 & 5 \\
A10 & 0.7 & 0.5 & 0 & 5 \\
\hline
\end{tabular}

This was followed by incubation using a detection system (EnVision+/HRP Rabbit, Dako) for $45 \mathrm{~min}$ at room temperature. Visualization by means of diaminobenzidine (DAB) was the next step (with a thermostat at $37{ }^{\circ} \mathrm{C}$ for 5 min). Nuclei were stained with hematoxylin and the specimens were then dehydrated with ethanol, highlighted with xylene and mounted on glass slides using mounting medium (Entelan TM). The positivity was again graded according to the number of detected fibers (Table 1). The numerical values from individual examinations were used in a statistical comparison of the treated (left femurs) and control group (right femurs).

\section{Statistical evaluation}

To analyse the data, we used both paired and unpaired tests. The non-parametric Wilcoxon matched-pairs test was used to evaluate whether there was any difference between the right limb without treatment and the left treated proximity in related parameters (longitudinal growth during experiment, difference in valgisation angle). To compare the proximities in categorical parameters (quality of cartilage healing, PAS, IHC, fluorescence, Pearls), the parameters were first binarized and the data were analysed using the McNemar matched-pairs test and the Fisher's exact test. The data were analysed using the IBM SPSS Statistics 19 for Windows (Release 19.0.0, IBM Corporation 2010).

\section{RESULTS}

The source of stem cells was bone marrow harvested from an unrelated animal, with successful aspiration of 10 $\mathrm{ml}$ in the case of most animals. Each transplant bore $2 \mathrm{x}$ $10^{6}$ MSCs. Immunophenotyping indicated more than $95 \%$ of the cells were positive for CD29, CD44 and CD90 and

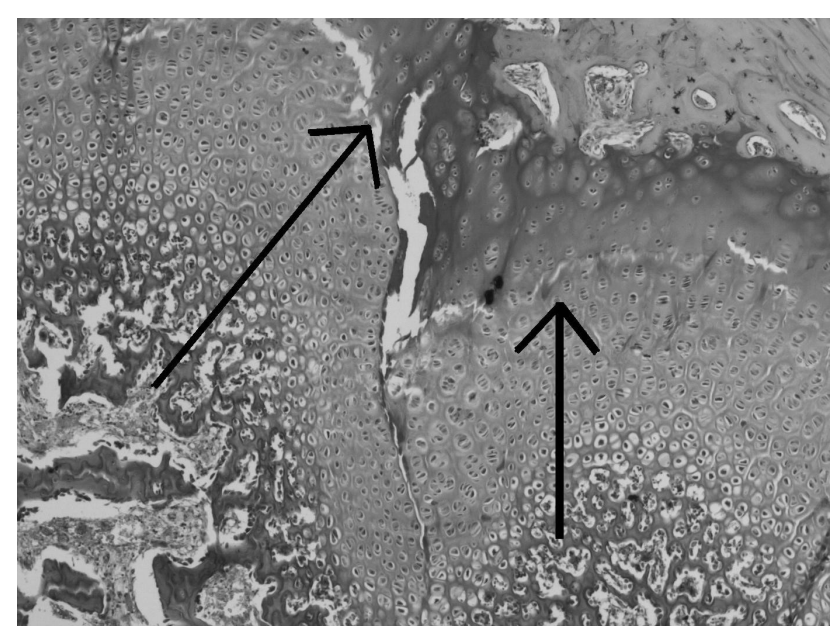

Fig. 2. Defect healing with newly-created hyaline cartilage with a sign of columnar formation, arrows pointing at the edge of defect, PAS staining, x40 magnification (animal A2 - left femur). 
Table 3. Quality of healed cartilage and type-II collagen detection in the growth plate defect.

\begin{tabular}{|c|c|c|c|c|}
\hline \multirow{2}{*}{ Animal } & \multicolumn{2}{|c|}{ Quality of new cartilage } & \multicolumn{2}{|c|}{ Immunohistochemistry - Col II } \\
\hline & Left femur & Right femur & Left femur & Right femur \\
\hline A1 & 0 & 0 & 2 & 1 \\
\hline A2 & 3 & 0 & 2 & 1 \\
\hline A3 & 0 & 0 & 1 & 1 \\
\hline A4 & 1 & 0 & 2 & 1 \\
\hline A5 & 3 & 0 & 3 & 0 \\
\hline A6 & 3 & 0 & 2 & 1 \\
\hline A7 & 3 & 0 & 2 & 1 \\
\hline A8 & 2 & 0 & 3 & 1 \\
\hline A9 & 3 & 0 & 2 & 1 \\
\hline A10 & 2 & 0 & 2 & 1 \\
\hline
\end{tabular}

they could be considered homogeneous. The expression of CD105 and CD147 was low. We obtained sufficient chondrocytes by taking the non-weight-bearing articular surface of distal femoral epiphysis.

The measurements of femur lengths showed that the left femur where combined scaffold with MSCs and $\mathrm{CHC}$ had been implanted in a physeal defect grew more in length $(0.71 \pm 0.11 \mathrm{~cm})$ than the right femur with a physeal defect without transplanted MSCs and CHC (0.55 $\mathrm{cm} \pm 0.2 \mathrm{~cm})(P=0.011)$. Angular (valgus) deformity in the left femur with MSCs and $\mathrm{CHC}$ transplanted in a physeal defect was $1.4^{\circ} \pm 0.7^{\circ}$. On the contrary, the right femur with a physeal defect without transplanted MSCs showed significantly greater valgus deformity $4.5^{\circ} \pm 1.0^{\circ}$ $(P=0.005)$ (Table 2).

Histological examination revealed that the defect in the left femur was filled with cartilage-like tissue in seven cases. In a half of all cases in treated group, direct signs indicating hyaline cartilage were found (Fig. 2) and the remaining cases showed at least signs indicating fibrocartilage. The average positivity evaluation was $2.0 \pm 1.0$. In the defect of the right femur, a bone bridge was found in all cases $(P=0.008)$. The newly-formed tissue underwent immunohistochemical testing using antibodies to detect type-II collagen, with positive results $(2.1 \pm 0.36)$ in nine cases in treated group. Examination of the newlycreated tissue in the defect of the right femur (control group) showed very low positivity for type-II collagen detection $(0.9 \pm 0.1 ; P=0.002$ - Table 3$)$. Fluorescent stain was detected in only 6 cases and only in trace amounts. Nevertheless, we are able to say that the chondrocytes were differentiated from the transplanted MSCs which had been previously stained with CM-DiI since in the control group no fluorochrome was detected $(P=0.031)$.

We found manipulation with the scaffold easy, without events of travelling from the defect or significantly changing its shape. Transplantation of this composite scaffold in combination with MSCs and chondrocytes led to the prevention of growth defect and angular deformity in the distal epiphysis of the femur. Compared to the control group (only a scaffold implanted), tissue similar to hyaline cartilage with signs of columnar organization typical of the growth plate occurred in most cases and favourably confirmed the presence of chondrocytes in differentiation in vivo with effect on stem cell differentiation.

\section{DISCUSSION}

Failure of bone growth after growth plate injury used to require invasive orthopaedic surgery with lengthening and external fixation ${ }^{9}$. Current experiments like the present study are in contrast, seeking to primarily prevent deformities and growth defects. The first studies dealt with implantation of cartilaginous or periosteal tissue in defects in the growth plate. In Wirth's study ${ }^{10}$ sheep tibia were used. A comparison of both materials favoured cartilaginous tissue over periost which led to quick growth of bone formations. The transplantation of chondrocyte grafts goes back to treatment of osteochondral lesions, particularly the use of mosaic arthroplasty. The method formerly referred to as promising, gradually became routine ${ }^{11-13}$. Foster et al. was probably the first to describe the method of transplanting chondrocytes in a defect in the growth plate in 1990. Authors of the experimental study on sheep, confirmed the method of filling the defect in the growth plate with autologous chondrocytes on a collagen scaffold. They described continuous proliferation of the chondrocyte culture, preservation of the cartilage-like structure and renewed architecture of the growth zone. The studies that followed tested different carriers of autologous chondrocytes - agarose ${ }^{14}$, atelocollagen ge ${ }^{15}$. The study carried out at our institution in 2002 used the miniature pig as a model and provided very good results ${ }^{16}$.

The study applied mesenchymal stem cells obtained from bone marrow (BMMC, BMMSCs), which has become the most frequent source of MSCs ( ref. $^{17,18}$ ). MSCs from periosteum or fat tissue ${ }^{18}$ tend to be less common.

One of the main problems in all similar methods is the transfer of cells into tissue in vivo. Moreover, in experimental animals it is not possible to achieve temporary immobilisation which is presumed in human traumatology. As a result, the potential of experiments shifts to the development and testing of new scaffolds. One of the 
goals of our study was to test a new scaffold which would be more resistant to mechanical load of bone and would provide increased support for the transferred cells. The tested product draws on the long-term research of our colleagues at the biotechnology laboratory of the Faculty of Chemistry, Brno University of Technology. The initial experiments employed a gel scaffold based on tissue adhesive ${ }^{17,19-21}$, and this scaffold form was unable to meet the requirements for resistance, let alone easy and simple surgical procedure. Subsequent studies used a threedimensional scaffold with self-supporting construction of collagen fibers ${ }^{22,23}$. Thanks to easy manipulation and favourable results achieved in the previous studies ${ }^{17,24,25}$, it was unnecessary to change the strategy fundamentally in terms of scaffold choice. However, further testing of cell viability was performed and scaffold architecture was reinforced to achieve higher rigidity and easier manipulation. In addition, the new scaffold construction made possible hydrodynamic seeding with MSCs and $\mathrm{CHC}$ which is a more effective method and one that provides more protection to transferred cells ${ }^{26}$. Another innovation was layering, with the periphery seeded with MSCs and the central part with chondrocytes.

Among the most frequently used differentiation factors in experiments in vitro and in vivo are TGF- $\beta 1$ (ref. ${ }^{18,27,28}$ ) and TGF- $\beta 3$ (ref. ${ }^{29}$ ). A member of the large family of TGF factors is also cartilage-derived morphogenetic protein 1 (CDMP1) which was successfully transferred to pig BMMSCs by gene transfer and induced their stimulation to chondrogenesis. The latest studies are searching for other factors that could be of use and one of these is the successfully tested chondroitin sulfate $\mathrm{C}$ (ref. ${ }^{30}$ ). The differentiation procedure we followed in our study ${ }^{3}$ proved useful in our previous experiments and it was further tested in other studies ${ }^{4}$.

A first questions arsing from the results, is possible applications in human medicine. It is only a matter of time before legalisation of the employed biocomposite will take place and made available in the treatment of pediatric injuries. Testing autologous transplantation seems the first and most reasonable step, followed by allogeneic transplantation. Despite this procedural obstacle, the first tests with human MSCs are already underway, with a current study in China describing promising regeneration of hyaline cartilage in mice and rabbits using human MSCs in combination with TGF- $\beta 3$ (ref. $\left.{ }^{31}\right)$.

\section{ACKNOWLEDGEMENTS}

This work was supported by the Ministry of Health of the Czech Republic (Research Projects of IGA MZ CR NR9896 - 3/2008) and Ministry of Education, Youth and Sport of the Czech Republic (NPV II 28061 30).

\section{REFERENCES}

1. Kim TK, Sharma B, Williams CG, Ruffner MA, Malik A, McFarland $E G$, Elisseeff $J H$. Experimentalmodel for cartilage tissue engineer- ing to regenerate the zonal organization of articular cartilage. Osteoarthritis Cartilage 2003;11:653-64.

2. Tisato V, Naresh K, Navarrete C, Dazzi F. Mesenchymal stem cells are effective at preventing but not at treating GvJD. Biology of blood and marrow transplantation 2007; 13:44-45.

3. Miura Y., Parvizi J., Fitzsimmons JS, O’Driscoll SW. Brief exposure to high-dose transforming growth factor-beta 1 enhances periosteal chondrogenesis in vitro: a preliminary report. J Bone Joint Surg 2002;84-A:793-9.

4. Gao J, Yao JQ, Caplan Al. Stem cells for tissue engineering of articular cartilage. Proc. IMechE 2007,221:441-50.

5. O'Driscoll SW, Keeley FW, Salter RB. Durability of regenerated articular cartilage produced by free autologous periosteal graft in major fullthickness defects in joint surfaces under the influence of continuous passive motion. J Bone Joint Surg. (Am) 1998;70:595.

6. Koshino T, Wada S, Ara Y, Saito T. Regeneration of degenerated articular cartilage after high tibial valgus osteotomy for medial compartmental osteoarthritis of the knee. Knee 2003,10:229-36.

7. Guo X, Wang CH, Zhang Y, Xia R, Hu M, Duan C, Zhao Q, Dong L, Lu J, Song YQ. Repair of Large Articular Cartilage Defects with Implants of Autologous Mesenchymal Stem Cells Seeded into Tricalcium Phosphate in a Sheep Model. Tissue Eng 2004;10:1818-29.

8. Lind M, Larsen A, Clausen C, Osther K, Everland H. Cartilage repair with chondrocytes in fibrin hydrogel and MPEG polylactide scaffold: an in vivo study in goats. Knee Surgery Sports traumatology arthroscopic 2008;16:690-8.

9. Jochymek J, Skvaril J. Ondrus S. Analysis of the Results of Bone Healing in Femurs Lengthened by the Gradual Distraction Method in Children and Adolescents. Acta Chir Orthop Traum Cech 2009;76:399-404

10. Wirth T, Byers S, Byard RW. The implantation of cartilaginous and periosteal tissue into growth plate defects. Int Orthop 1994;18:2208.

11. Peterson L, Brittberg M, Kiviranta I, Akerlund L, Lindahl A. Autologous chondrocyte transplantation. Biomechanics and long-term durability. Am J Sports Med 2002;30:2-12.

12. Makino T, Fujioka $H$, Kurosaka M, Matsui N, Yoshihara $H$, Tsonuda M Mizuno K. Histologie analysis of the implanted cartilage in an exactfit osteochondral transplantation model. Arthroscopy 2001;17:74751.

13. Ochi M, Uchio Y, Kawasaki K, Wakitani S, Iwasa J. Transplantation of cartilage-like tissue made by tissue engineering in the treatment of cartilage defects of the knee. J Bone Joint Surg Br 2002;84:571-8.

14. Lee EH, Gao GX, Bose K. Management of partial growth arrest: physis, fat, or silasties J Pediatr Orthop 1993;13:368-72.

15. Tobita M, Ochi M, Uchio Y, Mori R, Iwasa J, Katsube K, Motomura T. Treatment of growth plate injurywith autogenous chondrocytes: a study in rabbits. Acta Orthop Scand 2002;73:352-8.

16. Gal P, Adler J, Teyschl O, Fabian P, Biborva S, Necas A. Transplantation of the Autogenous Chondrocyte Graft to Physeal Defects: An Experimental Study in Pigs. Acta Vet Brno 2002;71:327-32.

17. Planka L, Gal P, Necas A, Kecova H, Filova E, Kren L, Krupa P. Prevention of bone bridge formation using transplantation of the autogenous mesenchymal stem cells to physeal defects: An experimental study in rabbits. Acta Vet Brno 2007;76:253-63.

18. Hui JHP, Li L, Teo YH, Ouyang HW, LEE EH. Comparative study of the ability of mesenchymal stem cells derived from bone marrow, periosteum, and adipose tissue in treatment of partial growth arrest in rabbit. Tissue engineering 2005;11:904-12.

19. Ahn Jl, Canale TS, Butler SD, Hasty KA. Stem cell repair of physeal cartilage. Journal of Orthopaedic Research 2004;22:1215-21.

20. Filova E, Rampichova M, Handl M, Lytvynets A, Halouzka R, Usvald D, Hlucilova J, Prochazka R, Dezortova M, Rolencova E, Trc T, Stastny E, Kolacna L, Hajek M, Motlik J, Amler E. Composite hyaluronate-type I collagen-fibrin scaffold in the therapy of osteochondral defects in miniature pigs. Physilogical research 2007;56:S5-S16.

21. Rampichova M, Filova E, Varga F, Lytvynets A, Prosecka E, Kolacna L, Motlik J, Necas A, Vajner L, Uhlik J, Amler E. Fibrin/Hyaluronic Acid Composite Hydrogels as Appropriate Scaffolds for In vivo Artificial Cartilage Implantation. Asaio Journal 2010;56:563-8.

22. Slovikova A, Vojtova L, Jancar J. Preparation and modification of collagen-based porous scaffold for tissue engineering. Cheemical Papers 2008;62:417-22. 
23. Jancar J, Slovikova A, Amler E, Krupa P, Kecova H, Planka L, Gal P, Necas A. Mechanical response of porous scaffolds for cartilage engineering. Physiological research 2007;56:S17-S25.

24. Gal P, Necas A, Planka L, Kecova H, Kren L, Krupa P, Hlucilova J, Usvald D. Chondrocytic potential of allogenic mesenchymal stem cells transplanted without immunosuppression to regenerate physea defect in rabbits. Acta Vet Brno 2007;76:265-75.

25. Planka L, Necas A, Srnec R, Rauser P, Stary D, Jancar J, Amler E, Filova E, Hlucilova J, Kren L, Gal P. Use of allogenic stem cells for the prevention of bone bridge formation in miniature pigs. Physiological Research 2009;58:885-93.

26. Jancar J, Vojtova L, Necas A, Srnec R, Urbanova L, Crha M. Stability of Collagen Scaffold Implants for Animals with latrogenic Articular Cartilage Defects. Acta Veterinaria Brno 2009;78:643-U107.

27. Lisignoli G, Cristino S, Piacentini A, Toneguzzi S, Grassi F, Cavallo C, Zini N, Solimando L, Maraldi NM, Facchini A. Cellular et molecular events during chondrogenesis of human mesenchymalstro- mal cells grown in three- dimensional hyaluronan based scaffold. Biomaterials 2005;26:5677-86.

28. Li WJ, Tuli R, Okafor C, Derfoul A, Danielson KG, Hall DJ, Tuan RS. A three-dimensional nanofibrous scaffold for cartilage tissue engineering using human mesenchymal stem cells. Biomaterials 2005;26:599-609.

29. Mackay AM, Beck SC, Murphy JM, Barry FP, Chichester CO, Pittenger MF. Chondrogenic differentiation of cultured human mesenchymal stem cells from marrow. Tissue Eng 1998;4:415-28.

30. Chen JL, Yin Z, Shen WLA, Chen X, Heng BC, Zou XH, Ouyang HW. Efficacy of hESC-MSCs in knitted silk-collagen scaffold for tendon tissue engineering and their roles. Biomaterials 2010;31:9438-51.

31. Park JS, Yang HN, Woo DG, Jeon SY, Park KH. Chondrogenesis of human mesenchymal stem cells in fibrin constructs evaluated in vitro and in nude mouse and rabbit defects models. Biomaterials 2001;32:1495-507. 